Check for updates

Cite this: Chem. Commun., 2021,

57, 2523

Received 10th November 2020

Accepted 31st January 2021

DOI: $10.1039 / \mathrm{dOcc0} 07393 \mathrm{~h}$

rsc.li/chemcomm

\title{
Post-synthetic modification of a highly flexible 3D soft porous metal-organic framework by incorporating conducting polypyrrole: enhanced MOF stability and capacitance as an electrode material $\dagger$
}

\author{
Zhen Li, Julio Fraile, Clara Viñas, (D) Francesc Teixidor (D) and José G. Planas (DD*
}

\begin{abstract}
Post-synthetic modification of a dynamic carborane-based soft porous crystal ( $1 \supset \mathrm{DMF}$ ) by in situ polymerization of pyrrole to polypyrrole (PPy) provided a permanently porous MOF/polypyrrole hybrid composite material that did not show the dynamic behavior of the mother MOF. Apart from stability, the introduction of conductive PPy in the composite material brings new properties to the otherwise non-conductive MOF.
\end{abstract}

Porous coordination polymers (CPs) or metal-organic frameworks (MOFs) have been widely studied as functional materials for a wide range of applications. ${ }^{1-5}$ More recently, MOF-based materials, including pristine MOFs and MOF composites have also attracted interest in the field of electrochemical energy storage and conversion. ${ }^{6-10}$ Since MOF discovery, much effort has been made to assure structural rigidity of MOFs as a way to maintain their porosity. In recent years, however, an intriguing aspect of MOFs has emerged, namely flexibility. ${ }^{11-17}$ Flexible MOFs, also known as third generation or "soft porous crystals" $(\mathrm{SPCs})^{17}$ are bistable or multistable crystalline materials with long range structural ordering and reversible transformability between states. The porosity of these SPCs is usually blocked by the guest solvent molecules that stabilize the framework and after removal, a phase transition to a nonporous phase is observed. Thus, flexibility often comes with the penalty of reduced stability, and loss of porosity is often the case. ${ }^{18}$ The work of Uemura and co-workers pioneered the stabilization of the otherwise nonporous soft crystals by the insertion of polystyrene into their pores. ${ }^{19}$ The polymeric guests prevent the spontaneous collapse of the framework and result in new and stable porous phases. This strategy has also been used for stabilizing mesoporous MOFs and thus preserving the porosity

Institut de Ciència de Materials de Barcelona, ICMAB-CSIC, Campus de la UAB, Bellaterra 08193, Spain. E-mail: jginerplanas@icmab.es

$\dagger$ Electronic supplementary information (ESI) available: Experimental methods, electrochemical measurements, IR, TGA data, PXRD patters, SEM images, CV curves, specific capacitance and impedance spectra. See DOI: 10.1039/ docc07393h upon activation. ${ }^{20}$ Polymerization of monomers inside the pores of MOFs is well known as a simple and convenient method for the hybridization of polymers and porous materials. $^{21-23}$ For example, Kitagawa and coworkers have demonstrated inclusion of typical vinyl polymers, polypyrrole (PPy) and polythiophene (PTh), into suitable nanochannels of various MOFs. ${ }^{24-27}$ Among the typical conductive polymers, polypyrrole (PPy) has acted as an excellent electrode material for supercapacitors ${ }^{28-30}$ and it has been used to overcome the poor electrical conductivity of MOFs and promote their applications in the fields of electrochemical energy storage and supercapacitors. ${ }^{7-9,31}$

Inspired by previous work on the stabilization of $\operatorname{SPCs}^{19}$ we hypothesize that inserting a functional polymer guest into these materials could modify the porous phase of the host, generating porosity, and at the same time the polymer could add new properties to the MOF composite. In pursuit of synthesizing water resistant and dynamic MOFs, we previously reported soft porous crystals (SPC) that behave as a crystalline sponge for a variety of guests $\left[\mathrm{Co}_{3}(\mathrm{BTB})_{2}(m \mathrm{CB})_{2}\right] \cdot$ Guest $(1 \supset$ Guest; BTB: 1,3,5-benzenetricarboxylate, $m \mathrm{CB}$ : 1,7-bis $\{$ (piridin4-yl)methanol\}-1,7-dicarba-closo-dodecaborane; Fig. 1). The presence of flexible mCB ligands introduces flexibility and water stability in the 3D structure of $\mathbf{1} \supset$ Guest, which shows a crystalline sponge behavior. ${ }^{18,32}$ However, the 3D structure of $1 \supset$ Guest is only stable when the 1D channels of this MOF are occupied with DMF or aromatic solvents. When these solvents
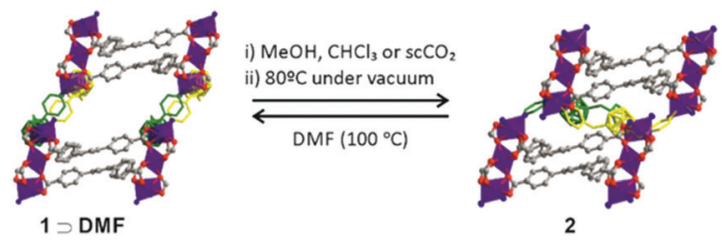

Fig. 1 Illustration of the reversible phase transformation between $1 \supset$ Guest and 2. 
are replaced by poor hydrogen bond acceptors (e.g., $\mathrm{MeOH}$, $\mathrm{CHCl}_{3}$ ) or removed by thermal heating under vacuum, a solid phase transition occurs to form the 2D non-porous structure 2 (Fig. 1). Although the process is reversible, the instability of the 3D phase (1) precludes its application, as porosity remains only in the presence of stabilizing guest solvent molecules. Taking advantage of the porous crystal behaviour of $1 \supset$ Guest, we now introduce pyrrole monomers into its pores and then initiate a chemical polymerization in order to (1) stabilize the 3D structure of 1 so that it can be permanently porous and (2) increase the electrical conductivity of the prepared composites. While these strategies have been previously reported independently, our report presents the first time that both strategies have been implemented at the same time and in a highly flexible and dynamic MOF.

Previous work reported that pyrrole (Py) can be polymerized to polypyrrole (PPy) within MOFs. ${ }^{24-26}$ Large 1D channel dimensions of $1(\sim 11 \times 13 \AA$ in $1 \supset$ DMF, Fig. 1$)$ are, in principle, suitable for such polymerization. To synthesize the composite, the as made $1 \supset$ DMF was firstly immersed into neat pyrrole for three days at $4{ }^{\circ} \mathrm{C}$ under static conditions. Pyrrole (Py) was changed once a day and the excess was removed. Loading of Py monomers into $\mathbf{1}(\mathbf{1} \supset$ Py thereafter) was firstly confirmed by IR. The IR spectrum of $\mathbf{1} \supset$ Py showed the disappearance of the DMF signals and appearance of representative bands of Py (Fig. S1, ESI $\dagger$ ). No obvious colour change was observed from $1 \supset \mathbf{D M F}$ to $\mathbf{1} \supset \mathbf{P y}$, both being red and crystalline (Fig. S1, ESI $\dagger$ ). Thermogravimetric analysis (TGA) for $\mathbf{1} \supset$ Py showed a loading of 6 Py monomers per formula unit (Fig. S2, ESI $\dagger$ ). The obtained $1 \supset$ Py was then treated with a mild oxidant $\left(\mathrm{K}_{2} \mathrm{~S}_{2} \mathrm{O}_{8}\right)$ as an auxiliary oxidizing agent, for the in situ polymerization of Py. ${ }^{33-35}$ Accordingly, freshly made $\mathbf{1} \supset$ Py crystals were soaked in a $0.2 \mathrm{M} \mathrm{K} \mathrm{K}_{2} \mathrm{~S}_{2} \mathrm{O}_{8}$ aqueous solution at room temperature for $24 \mathrm{~h}$ to provide a black colored composite of 1 with PPy (1 つ PPy; Fig. 2 and Fig. S1, ESI $\dagger$ ). Shorter reaction times resulted in lower polymer contents and thus stabilization of $\mathbf{1}$ could not be achieved (vide infra). PPy contents in $1 \supset$ PPy was determined to be $c a .26 \%$ by TGA (Fig. S3, ESI $\dagger$ ). IR shows the disappearance of Py monomer in $1 \supset$ PPy (Fig. S1a and b, ESI $\dagger$ ) and the black color of $\mathbf{1} \supset$ PPy evidenced the presence of PPy in the composite crystals. A comparison of the PXRD patterns

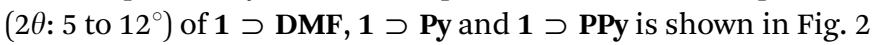
(see complete PXRD patterns in Fig. S4, ESI $\dagger$ ). $1 \supset$ DMF is a 3D structure composed of $2 \mathrm{D}$ rigid $\mathrm{Co} / \mathrm{BTB}$ layers and flexible carborane pillars (Fig. 1). The Co/BTB layers are on the (100) plane and the carborane pillars are on the (001) plane (Fig. S5a, $\mathrm{ESI} \dagger)$, with interlayer distances of $13.8\left(2 \theta 6.46^{\circ}\right)$ and $13.2 \AA$ $\left(2 \theta 6.68^{\circ}\right)$, respectively (Table S1, ESI $\dagger$ ). The incorporation of Py into the channels of 1 provokes a slight increase of the (001) interlayer distance (13.7 $\AA$; $2 \theta 6.42^{\circ}$, Fig. 2), consistent with the observed breathing effect of host 1 to guest adsorption (Fig. S5b, ESI $\dagger$ ). However, the polymerization of Py to PPy in 1 provokes a significant change in the PXRD pattern that shows a displacement of the diffraction peak up to $2 \theta 5.6^{\circ}(15.6 \AA)$ in $1 \supset$ PPy. The larger interlayer distance, corresponding to the low angle PXRD peaks in these materials, has significantly
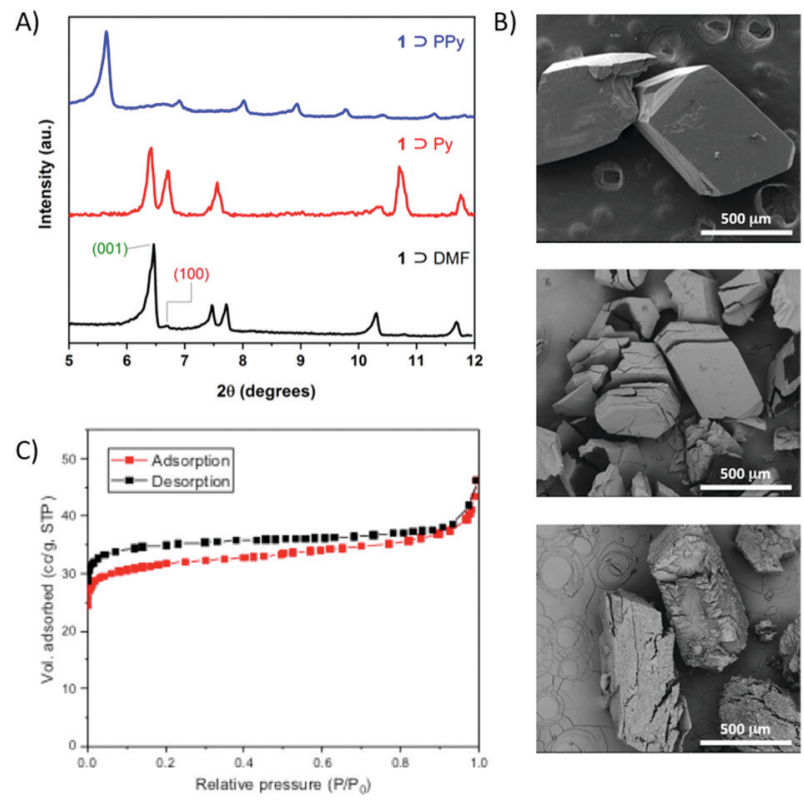

Fig. 2 PXRD data (A) and Scanning Electron Microscopy (SEM) images (B) of $1 \supset$ DMF, $1 \supset$ Py and $1 \supset$ PPy from top to bottom. (C) $\mathrm{N}_{2}$ adsorption-desorption isotherms of $1 \supset$ PPy.

expanded from $13.8 \AA$ in $\mathbf{1} \supset$ Py to $15.6 \AA$ in $\mathbf{1} \supset$ PPy. This displacement is consistent with the swelling of the unit cell parameters of $\mathbf{1}$ on polymerization of Py into its pores. No such swelling was observed when $1 \supset$ DMF was treated with $\mathrm{K}_{2} \mathrm{~S}_{2} \mathrm{O}_{8}$ under the same conditions (Fig. S6, ESI $\dagger$ ). Unfortunately the composite crystals did not diffract well, even under synchrotron radiation.

The morphology of the crystals in SEM images of $1 \supset$ DMF and $1 \supset$ Py is consistent with the above PXRD data. The assynthesized $1 \supset$ DMF (top of Fig. 2B) consists of well-defined block shaped crystals with smooth surfaces and with dimensions of $c a$. $500 \mu \mathrm{m}$. Crystals of a similar shape, size and smooth surface are observed in the Py exchanged $1 \supset$ Py crystals (middle of Fig. 2B). Compared with the latter two, SEM images for the composite material $1 \supset$ PPy show a much rougher surface (bottom of Fig. 2 and Fig. S7, ESI $\dagger$ ). Consistently with the PXRD data (Fig. 2A) the polymerization of Py within the channels of $\mathbf{1}$ provokes a different crystalline phase with a larger cell volume than that of $\mathbf{1} \supset$ DMF or $\mathbf{1} \supset$ Py. The increase in the unit cell parameters in $\mathbf{1} \supset$ PPy is large enough to provoke cracks as a result of the long-range strain introduced when the internal periodicity is disrupted (Fig. 2B). SEM images of $1 \supset$ PPy show that the crystal fragments still exhibit a similar morphology to those in $\mathbf{1} \supset$ DMF or $\mathbf{1} \supset$ Py but now the crystals are fully covered by layers of PPy. Partial digestion of the MOF composite by ammonium hydroxide or crashing the crystals show that the 2D PPy layers are not only on the surface but also embedded within the crystals (Fig. S7 and S8, ESI $\dagger$ ). The data suggest that the Py monomers in the pores of $\mathbf{1}$ are effectively polymerized into PPy that grows within the MOF network. Complete removal of the host framework of $1 \supset$ PPy in a solution of ammonium hydroxide allowed the isolation of the 
intercalated PPy, which was fully characterized using SEM and IR (Fig. S9, ESI $\dagger$ ). The morphology of the PPy isolated from $1 \supset$ PPy correlates well with a 2D thin morphology as that reported by Kitagawa and coworkers. ${ }^{24}$

As mentioned above, the 3D structure of $\mathbf{1} \supset$ DMF transforms into the non porous 2D structure 2 when immersed in $\mathrm{MeOH} .{ }^{18}$ Thus, in order to evaluate the positive effect of the polymer in stabilizing the structure of the composite, we immersed $1 \supset$ PPy in excess methanol for 5 days and further dried it at $90{ }^{\circ} \mathrm{C}$ for $12 \mathrm{~h}$. The PXRD results clearly revealed that no evidence for phase transformation can be found after methanol treatment and drying, confirming the crucial role of the polymer in stabilizing the porous architecture of $\mathbf{1} \supset$ PPy (Fig. S10, ESI $\dagger$ ). Sorption measurements revealed that $\mathbf{1} \supset \mathbf{P P y}$ is porous to $\mathrm{N}_{2}$ at $77 \mathrm{~K}$ and $1 \mathrm{bar}$, and the BET surface area was found to be $124 \mathrm{~m}^{2} \mathrm{~g}^{-1}$ (Fig. 2C). The pore size distribution was calculated based on the Barrett-Joyner-Halenda (BJH) model from the adsorption curve (Fig. S11A, ESI $\dagger$ ). Interestingly, the micropores from the MOFs with a diameter of $1.79 \mathrm{~nm}$ were still maintained, which is in good agreement with the adsorption-desorption isotherms. Mesopores (4.10 and $11.69 \mathrm{~nm}$ ), probably corresponding to the interparticle pores in $\mathbf{1} \supset \mathbf{P P y}$, were also observed. PXRD measurement after the sorption experiments provides further proof of stability for $\mathbf{1} \supset$ PPy (Fig. S11B, ESI $\dagger$ ).

Having determined that $\mathbf{1} \supset \mathbf{P P y}$ is robust and permanently porous, and considering the good conductivity of PPy, we then evaluated the performance of the $\mathbf{1} \supset$ PPy composite as an electrode material for supercapacitors (SCs). Low conductivity of MOFs is still a key factor that prevents them from achieving high electrochemical performance. ${ }^{9,10}$ As a control, we have also evaluated the electrochemical performance of $\mathbf{1} \supset$ DMF. The preparation of the electrode is briefly described here and the specific details are given in Fig. S12 (ESI $\dagger$ ). First, crystals of $1 \supset$ DMF were grown onto a piece $(1 \mathrm{~cm} \times 1 \mathrm{~cm})$ of PPy coated nickel foam (NF@PPy). Conductive PPy on the NF substrate allows effective nucleation and good adhesion of $\mathbf{1} \supset$ DMF on the substrate. ${ }^{36}$ Following the above procedure, DMF molecules in the obtained composite $(\mathbf{1} \supset$ DMF/NF@PPy or 1/DMF; Fig. 3A and Fig. S13, ESI $\dagger$ ) were then replaced by Py (providing $1 \supset$ Py/NF@PPy or 1/Py) and finally polymerized by $\mathrm{K}_{2} \mathrm{~S}_{2} \mathrm{O}_{8}$ to obtain the desired $\mathbf{1} \supset \mathbf{P P y} / \mathrm{NF} @ P P y(\mathbf{1} / \mathbf{P P y})$ composite electrode material (Fig. 3B and Fig. S13, ESI $\dagger$ ). The electrochemical performance of the composites (1/PPy and $\mathbf{1} / \mathbf{D M F})$ was tested as electrode materials for supercapacitors using cyclic voltammetry (CV; Fig. 3C) and galvanostatic charge-discharge technique is (using) three-electrode system (GCD; Fig. S14, ESI $\dagger$ ). The CV results show that the synthesized $\mathbf{1} / \mathbf{P P y}$ composite exhibits a much better electrochemical performance than 1/DMF. Fig. 3C shows the representative $\mathrm{CV}$ scans of both samples performed at a scan rate of $5 \mathrm{mV} \mathrm{s}^{-1}$ in $1.0 \mathrm{M} \mathrm{Na}_{2} \mathrm{SO}_{4}$ aqueous solution. The 1/PPy composite produces larger currents than 1/DMF, indicating that the incorporation of PPy into the MOF structure clearly enhances the electrochemical performance of $\mathbf{1} / \mathbf{P P y} .{ }^{37}$ To further explore the electrochemical properties, we measured the $\mathrm{CV}$ curves of 1/PPy and 1/DMF composites at different scan rates
A)
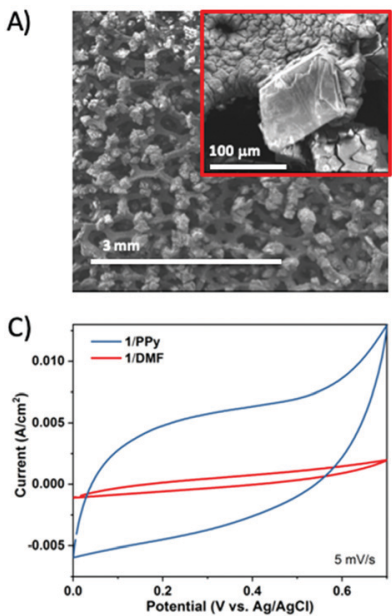

B)

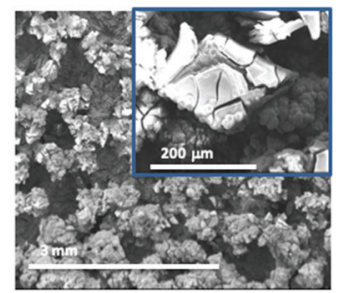

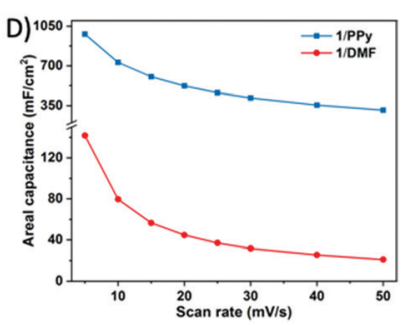

Fig. 3 Scanning Electron Microscopy (SEM) images of 1/DMF (A) and 1/PPy (B); inset images show the composite materials grown on polypyrrole coated nickel foam substrates. CV curves of 1/DMF and 1/PPy composites at a rate of $5 \mathrm{mV} \mathrm{s}^{-1}$ with potential from 0.0 to $0.7 \mathrm{~V}$ (C). Areal capacitance versus scan rate for both composites (D).

(Fig. S14A and B, ESI $\dagger$ ). The curves exhibit a distorted rectangular shape with a mirror-image feature, suggesting a reasonable capacitance behaviour for $\mathbf{1} / \mathbf{P P y}$. In addition, the shape of the CV curves does not significantly change with the increase in the scan rates, which indicates the low resistance of the electrode. ${ }^{38}$ Consistently, the calculated specific capacitance $(C)$ values of 1/PPy are much higher than those of $\mathbf{1 / D M F}$ at scan rates ranging from 5 to $50 \mathrm{mV} \mathrm{s}^{-1}$ (Fig. 3D). At $50 \mathrm{mV} \mathrm{s}^{-1}, \mathbf{1} / \mathbf{P P y}$ retains $c a$. $32 \%$ of its original capacitance, while $\mathbf{1} / \mathbf{D M F}$ only retains $15 \%$. The capacitance contribution from the NF@PPy or PPy/NF@PPy substrates was found to be negligible $\left(6.6\right.$ or $7.9 \mathrm{mF} \mathrm{cm}^{-2}$, respectively, at $5 \mathrm{mV} \mathrm{s}^{-1}$; Fig. S15, ESI $\dagger$ ). Calculated areal capacitance values of 1/PPy from GCD curves (Fig. S14D, ESI $\dagger$ ) are comparable to those obtained from the CV curves. Thus, not only higher capacitance but also better capacitance retention can be clearly observed for 1/PPy compared to $\mathbf{1 / D M F}$, further confirming that the electrochemical performance of the MOF has been enhanced by incorporating polypyrrole. Electrochemical impedance spectroscopy (EIS) measurements of 1/PPy and 1/DMF (Fig. S16, ESI $\dagger$ ) indicate again the role of the PPy in enhancing the electrical conductivity of the material. The cycling performance of the as-prepared electrodes was further characterized by CV measurements at a scan rate of $5 \mathrm{mV} \mathrm{s}^{-1}$ (Fig. S16, ESI $\dagger$ ). The working electrode of the 1/PPy composite exhibited an excellent cycling capability compared to $\mathbf{1} / \mathbf{D M F}$, with no sudden loss in capacitance up to 1000 cycles (Fig. S17, ESI $\dagger$ ) with the 3D structure remaining stable (Fig. S18, ESI $\dagger$ ). On the contrary, the capacitance of 1/DMF drops sharply in the first 50 cycles (Fig. S17, ESI $\dagger$ ) and the structure transformed to non porous 2D phases (Fig. S18, ESI $\dagger$ ). Thus, the stability of the $\mathbf{1} \supset$ PPy composite in the electrode proves that the porous structure of this new composite was maintained during the chargedischarge processes and confirms the positive effect of the PPy in stabilizing the porous structure of flexible and dynamic MOFs. 
In conclusion, a highly flexible carborane-based MOF (1 つ DMF) was employed for the in situ polymerization of pyrrole (Py) to polypyrrole (PPy). In the absence of stabilizing $\mathrm{DMF}$, the 3D structure of $\mathbf{1}$ is not stable and suffers a solid phase transition leading to a $2 \mathrm{D}$ non porous structure. We report that DMF can be easily replaced by Py in $1 \supset$ DMF providing $\mathbf{1} \supset \mathbf{P y}$, which maintains the 3D structure and slightly increased the cell parameters. Chemical polymerization of the pyrrole units takes place in the pores of $\mathbf{1}$ and provides another new stable porous phase $1 \supset$ PPy. The new MOF/ polymer hybrid composite material is stable and does not show the flexible behaviour of the parent MOF ( $\supset$ DMF). The introduction of conductive PPy in the structure of $\mathbf{1}$ brings new properties to the otherwise non conductive MOF. As a proof of concept for potential applications, we have evaluated the performance of the $\mathbf{1} \supset$ PPy composite as the electrode material for supercapacitors (SCs) and compared it with that of the original $1 \supset$ DMF. The electrochemical results indicate a clear improvement in charge storage ability, rate capability and cycling stability of the MOF/polymer hybrid composite. The specific capacitance of $\mathbf{1 / P P y}$ is improved seven-fold compared to the non hybridized 1/DMF.

The present work demonstrates how new MOF polymer hybrids can be prepared, through the combination of in situ polymerization and highly flexible and dynamic MOFs such as soft porous crystals or even those potentially porous MOFs that are not stable under conventional activation conditions.

This work was financially supported by MINECO (CTQ201675150-R, PID2019-106832RB-I00) and the Generalitat de Catalunya (2017/SGR/1720). ICMAB acknowledges the support of the Spanish MINECO (Grant SEV-2015-0496). Z. Li is enrolled in the UAB PhD program and acknowledges the China Scholarship Council (CSC) for his PhD grants (201808310071). Z. Li thanks Fangchang Tan and Lei Gan for their assistance with the synthesis of $1 \supset$ DMF. The present publication is dedicated to Prof. Alan Welch on the occasion of his retirement from Heriot-Watt University.

\section{Conflicts of interest}

There are no conflicts to declare.

\section{Notes and references}

1 Y. Tang and S. Tanase, Microporous Mesoporous Mater., 2020, 295, 109946

2 S. Rojas and P. Horcajada, Chem. Rev., 2020, 120, 8378-8415.

3 A. Bavykina, N. Kolobov, I. S. Khan, J. A. Bau, A. Ramirez and J. Gascon, Chem. Rev., 2020, 120, 8468-8535.

4 G. Mínguez Espallargas and E. Coronado, Chem. Soc. Rev., 2018, 47, 533-557.

5 Ü. Kökcam-Demir, A. Goldman, L. Esrafili, M. Gharib, A. Morsali, O. Weingart and C. Janiak, Chem. Soc. Rev., 2020, 49, 2751-2798.

6 C.-W. Kung, K. Otake, C. T. Buru, S. Goswami, Y. Cui, J. T. Hupp, A. M. Spokoyny and O. K. Farha, J. Am. Chem. Soc., 2018, 140, 3871-3875.
7 K. Zhang, K. O. Kirlikovali, Q. Van Le, Z. Jin, R. S. Varma, H. W. Jang, O. K. Farha and M. Shokouhimehr, ACS Appl. Nano Mater., 2020, 3, 3964-3990.

8 M. Kalaj, K. C. Bentz, S. Ayala, J. M. Palomba, K. S. Barcus, Y. Katayama and S. M. Cohen, Chem. Rev., 2020, 120, 18267-18302.

9 Z. Liang, C. Qu, W. Guo, R. Zou and Q. Xu, Adv. Mater., 2018, 30, e1702891.

10 M. Du, Q. Li, Y. Zhao, C.-S. Liu and H. Pang, Coord. Chem. Rev., 2020, 416, 213341.

11 K. Uemura, R. Matsuda and S. Kitagawa, J. Solid State Chem., 2005, 178, 2420-2429.

12 J. H. Lee, S. Jeoung, Y. G. Chung and H. R. Moon, Coord. Chem. Rev., 2019, 389, 161-188.

13 W. W. Fan, Y. Cheng, L. Y. Zheng and Q. E. Cao, Chemistry, 2020, 26, 2766-2779.

14 S. K. Elsaidi, M. H. Mohamed, D. Banerjee and P. K. Thallapally, Coord. Chem. Rev., 2018, 358, 125-152.

15 R. E. Morris and L. Brammer, Chem. Soc. Rev., 2017, 46, 5444-5462.

16 G. Férey, New J. Chem., 2016, 40, 3950-3967.

17 S. Horike, S. Shimomura and S. Kitagawa, Nat. Chem., 2009, 1, 695-704.

18 F. Tan, A. López-Periago, M. E. Light, J. Cirera, E. Ruiz, A. Borrás, F. Teixidor, C. Viñas, C. Domingo and J. G. Planas, Adv. Mater., 2018, 30, 1800726.

19 B. Le Ouay, S. Kitagawa and T. Uemura, J. Am. Chem. Soc., 2017, 139, 7886-7892.

20 L. Peng, S. Yang, S. Jawahery, S. M. Moosavi, A. J. Huckaba, M. Asgari, E. Oveisi, M. K. Nazeeruddin, B. Smit and W. L. Queen, J. Am. Chem. Soc., 2019, 141, 12397-12405.

21 T. Uemura, S. Horike and S. Kitagawa, Chem. - Asian J., 2006, 1, $36-44$.

22 B. L. Ouay and T. Uemura, Isr. J. Chem., 2018, 58, 995-1009.

23 T. Kitao, Y. Zhang, S. Kitagawa, B. Wang and T. Uemura, Chem. Soc. Rev., 2017, 46, 3108-3133.

24 N. Yanai, T. Uemura, M. Ohba, Y. Kadowaki, M. Maesato, M. Takenaka, S. Nishitsuji, H. Hasegawa and S. Kitagawa, Angew. Chem., Int. Ed., 2008, 47, 9883-9886.

25 T. Uemura, Y. Kadowaki, N. Yanai and S. Kitagawa, Chem. Mater., 2009, 21, 4096-4098.

26 Q.-X. Wang and C.-Y. Zhang, Macromol. Rapid Commun., 2011, 32, $1610-1614$

27 M. W. A. MacLean, T. Kitao, T. Suga, M. Mizuno, S. Seki, T. Uemura and S. Kitagawa, Angew. Chem., Int. Ed., 2016, 55, 708-713.

28 S. Kuwabata, J. Nakamura and H. Yoneyama, J. Electrochem. Soc., 1990, 137, 2147-2150.

29 W. Ji, J. Ji, X. Cui, J. Chen, D. Liu, H. Deng and Q. Fu, Chem. Commun., 2015, 51, 7669-7672.

30 G. A. Snook, P. Kao and A. S. Best, J. Power Sources, 2011, 196, 1-12.

31 B. Dhara, S. S. Nagarkar, J. Kumar, V. Kumar, P. K. Jha, S. K. Ghosh, S. Nair and N. Ballav, J. Phys. Chem. Lett., 2016, 7, 2945-2950.

32 M. Y. Tsang, S. Rodríguez-Hermida, K. C. Stylianou, F. Tan, D. Negi, F. Teixidor, C. Viñas, D. Choquesillo-Lazarte, C. Verdugo-Escamilla, M. Guerrero, J. Sort, J. Juanhuix, D. Maspoch and J. G. Planas, Cryst. Growth Des., 2017, 17, 846-857.

33 K. C. Khulbe, R. S. Mann and C. P. Khulbe, J. Polym. Sci., Polym. Chem. Ed., 1982, 20, 1089-1095.

34 X. Zhang, J. Zhang, W. Song and Z. Liu, J. Phys. Chem. B, 2006, 110, 1158-1165.

35 M. Rahaman, A. Aldalbahi, M. Almoiqli and S. Alzahly, Polymers, $2018,10,632$.

36 R. Abazari, S. Sanati, A. Morsali, A. M. Z. Slawin, C. L. CarpenterWarren, W. Chen and A. Zheng, J. Mater. Chem. A, 2019, 7, 11953-11966.

$37 \mathrm{CV}$ measurement for polypyrrole coated nickel foam at $5 \mathrm{mV} \mathrm{s}^{-1}$ shows a negligable contribution of $6.6 \mathrm{mF} \mathrm{cm}^{-2}$ which proves that the electrode capacitance is mainly originated from the 1/PPy material.

38 Y. Yang, Q. Huang, L. Niu, D. Wang, C. Yan, Y. She and Z. Zheng, Adv. Mater., 2017, 29, 1606679. 